\title{
3D Heart Model and 4D Flow MRI 20 Years after Spiral Arterial Switch Operation
}

\author{
${ }^{1}$ Department of Cardiac and Thoracic Vascular Surgery, University \\ Hospital of Schleswig-Holstein, Campus Luebeck, Luebeck, \\ Schleswig-Holstein, Germany \\ 2 Kheradvar Research Group, Edwards Lifesciences Center for \\ Advanced Cardiovascular Technology, University of California, Irvine, \\ Irvine, California, United States \\ ${ }^{3}$ Department of Congenital Heart Disease and Pediatric Cardiology, \\ University Hospital of Schleswig-Holstein, Campus Kiel, Kiel, \\ Schleswig-Holstein, Germany \\ Thorac Cardiovasc Surg Rep 2016;5:44-46.
}

Hans-Hinrich Sievers ${ }^{1}$ Arash Kheradvar ${ }^{2}$ Hans-Heiner Kramer ${ }^{3}$ Carsten Rickers ${ }^{3}$

\begin{abstract}
Address for correspondence Hans-Hinrich Sievers, MD, Department of Cardiac and Thoracic Vascular Surgery, University Hospital of Schleswig-Holstein, Campus Luebeck, Ratzeburger Allee 160, Luebeck 23538, Germany (e-mail: hans-hinrich.sievers@uksh.de).
\end{abstract}

\author{
Abstract \\ Keywords \\ - transposition of great \\ arteries \\ - magnetic resonance \\ imaging \\ - physiological spiral \\ anastomosis
}

Case of a patient is presented here 20 years after spiral direct anastomosis of the great arteries in an arterial switch operation. Three-dimensional model of the heart combined with four-dimensional flow magnetic resonance imaging presents a novel comprehensive way to assess surgical results.

\section{Introduction}

The first successful arterial repair for transposition of the great arteries (TGAs) was performed in 1975 by Jatene et al using a direct spiral anastomosis of the great arteries. ${ }^{1}$ In 1981, the Lecompte et al technique, characterized by transposition of the pulmonary artery (PA) bifurcation in front of the ascending aorta, was introduced with excellent shortterm results. ${ }^{2}$ Nevertheless, this unphysiological anatomy is reported to contribute to dilatation of the ascending aorta proximal to the pulmonary bifurcation, progressive aortic regurgitation, steep angled aortic arch, and most commonly pulmonary stenosis. ${ }^{3,4}$ Little is known about the physiological spiral anastomosis of the great arteries as an alternative switch operation especially after somatic growth. We present morphology through a three-dimensional heart model and four-dimensional (4D) flow magnetic resonance imaging (MRI) in such a case.

received

March 4, 2016

accepted after revision

May 1, 2016

published online

June 21, 2016

\section{Case Description}

In 1991, a 2-day-old newborn male with simple TGA, normal coronary artery pattern (1L-2RCx), and an intermediate position of the great arteries (aortic root to pulmonary root between anterior-posterior and side-by-side position) underwent the arterial switch operation using spiral anastomoses of the great arteries and a pantaloon patch of untreated autologous pericardium to fill the coronary button holes and to extend the short main PA for $1.0 \mathrm{~cm}$ (-Fig. 1). In 2012, the patient underwent invasive catheterization showing a mild pulmonary stenosis (i.e., maximum gradient of $20 \mathrm{~mm} \mathrm{Hg}$ ) with normal function of semilunar valves. In 2015, a 3D model of his heart was developed out of polyurethane according to phase-contrast cardiac MRI data (- Fig. 2: MR voxel size $1.12 \times 1.11 \times 7 \mathrm{~mm}$; - Fig. 3: 3D model pixel size $0.66 \times 0.66 \mathrm{~mm}$, layer size $1 \mathrm{~mm}$ ) with a resolution of $\pm 0.3 \%$ and a layer thickness of $0.12 \mathrm{~mm}$ (Materialise $\mathrm{GmbH}$,

\footnotetext{
(c) 2016 Georg Thieme Verlag KG Stuttgart · New York
}

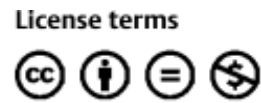




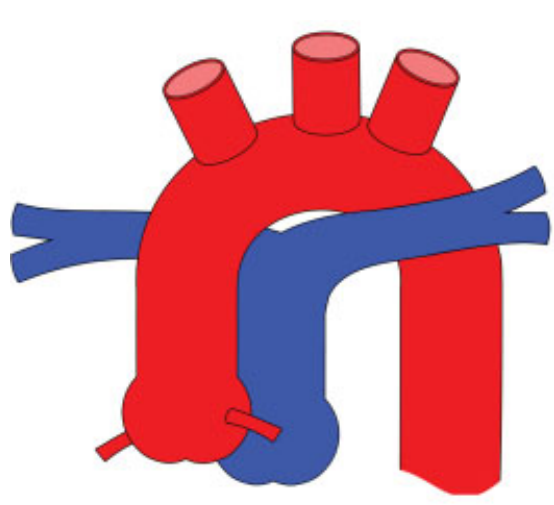

A. TGA

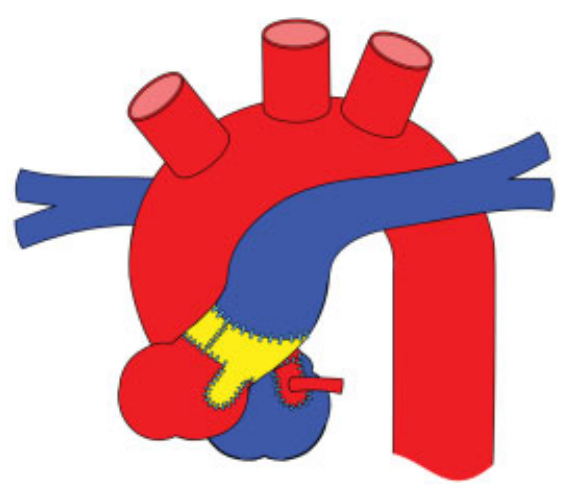

C. Spiral (postoperative)

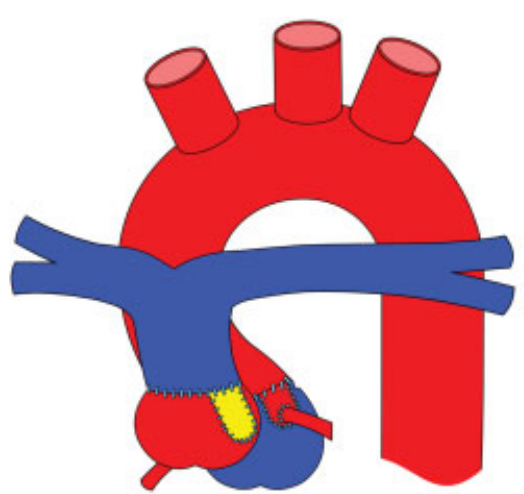

B. Lecompte

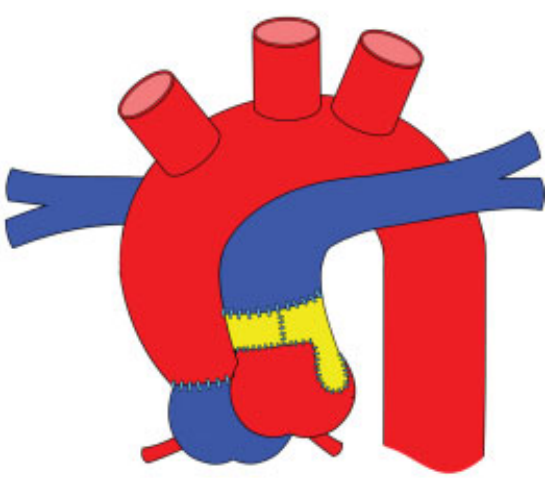

D. Spiral (20 years follow-up)

Fig. 1 Schematic drawing of the different operative techniques for anatomic correction of TGAs. (A) Most frequent anatomy of TGA with the aortic root on the right side of the pulmonary root. (B) Classical Lecompte et al technique with the pulmonary bifurcation transposed in front of the aorta. (C) Spiral anastomosis of the great arteries leaving the spiral physiological arrangement of the great arteries intact. (D) Follow-up situs 20 years after spiral anastomosis showing the spontaneous rotation of the neopulmonary (former aortic) root to the left of the neoaortic (former pulmonary) root approaching physiological optical conditions. Blue color, pulmonary; red color, aortic; TGA, transposition of the great artery; yellow color, pericardial patch for filling the coronary buttons and for elongation of the pulmonary artery.
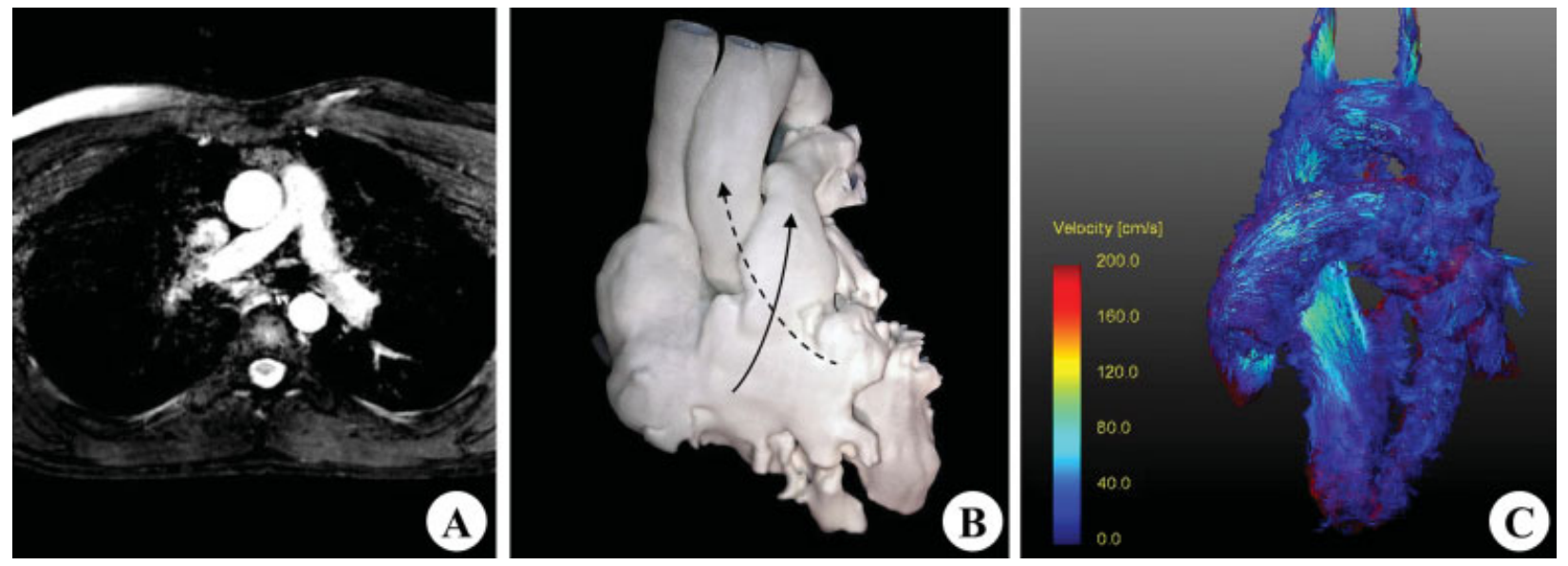

Fig. 2 (A) Axial cine MRI snapshot, (B) 3D whole heart model, (C) blood flow streamlines derived from 4D flow MRI. The spiral physiological arrangement of the great arteries, the rotation of the neopulmonary root to the left, and near-normal spiral flow along with a mild narrowing of the right pulmonary artery at the bifurcation are shown. Arrows in (B) indicate schematically the spiral flow vectors. Dotted arrow, flow from the left ventricle; solid arrow, flow from the right ventricle. 4D, four-dimensional; MRI, magnetic resonance imaging; 3D, three-dimensional. 


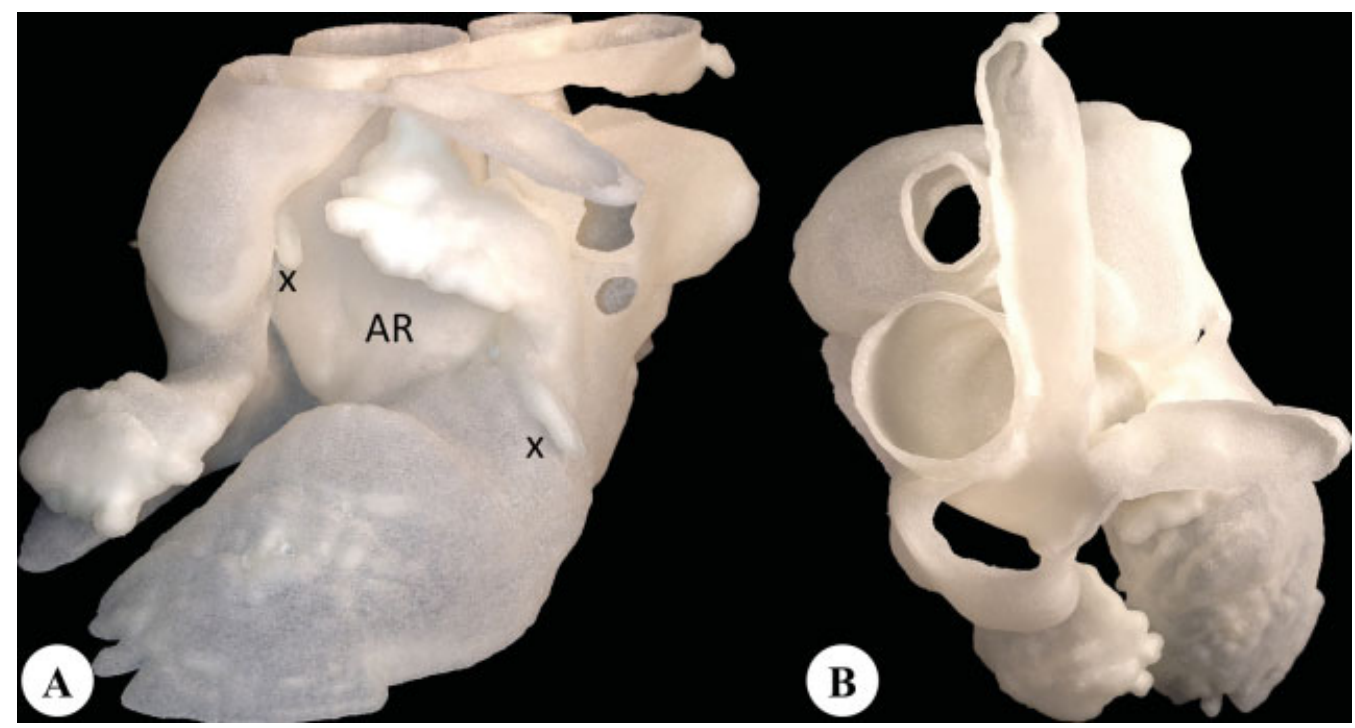

Fig. 3 (A) Left side and (B) top view of a 3D heart model shows the neoaortic root (AR in A), the leftward rotated neopulmonary root, the coronary arteries $(X)$, and the pulmonary bifurcation with right pulmonary artery curving around the ascending aorta. 3D, three-dimensional.

Gilching, Germany). At follow-up, the neopulmonary root was found to have spontaneously rotated from a preoperative rightward position to the left, and the physiological spiral anatomy of the great arteries and physiological blood flow were restored (-Fig. 2). A mild narrowing of the right PA behind the aorta (-Fig. 2A) was discovered. In - Fig. 3, the position of the left coronary artery between the neoaortic root and the neopulmonary root is shown as well as the pulmonary bifurcation in a cross-section. First pass myocardial perfusion MRI at rest and during stress confirmed a homogenous microcirculation of the left ventricle.

\section{Discussion}

The 3D heart model nicely shows the physiological spiral relations of the great arteries. Flow streamlines throughout the pulmonary and aortic outflow tracts were found parallel in a spiral fashion and apart from each other ( $\mathbf{F i g}$. 2C). The spontaneous rotation of the neopulmonary root to a leftward position was an unexpected finding and may probably indicate some morphological plasticity during growth. The right PA seems to be under some tension, which may have been improved if the distal PA anastomosis was performed somewhat into the left PA to gain some length for the right PA. The left anterior descending coronary artery was not found to be under compression. No significant stenosis was found at the area of the pericardial pantaloon tube patch confirming that fresh autologous pericardium is an appropriate material for PA patch plastics during arterial switch operation, as suggested by Paillole et al. ${ }^{5}$
We conclude that 3D heart models combined with 4D flow MRI are excellent means to assess the surgical results and visualize the spatial relationships of the anatomical structures.

\section{Acknowledgments}

The authors are grateful to Mrs. Traudel Hansen for her assistance in patient management and Mr. Michael Diwoky for his support in preparation of this report.

\section{References}

1 Jatene AD, Fontes VF, Paulista PP, et al. Successful anatomic correction of transposition of the great vessels. A preliminary report. Arq Bras Cardiol 1975;28(4):461-464

2 Lecompte Y, Zannini L, Hazan E, et al. Anatomic correction of transposition of the great arteries. J Thorac Cardiovasc Surg 1981; 82(4):629-631

3 Losay J, Touchot A, Capderou A, et al. Aortic valve regurgitation after arterial switch operation for transposition of the great arteries: incidence, risk factors, and outcome. J Am Coll Cardiol 2006;47(10):2057-2062

4 Rickers C, Kheradvar A, Sievers HH, et al. Is the Lecompte technique the last word on transposition of the great arteries repair for all patients? A magnetic resonance imaging study including a spiral technique two decades postoperatively. Interact Cardiovasc Thorac Surg 2016;22(6):817-825

5 Paillole C, Sidi D, Kachaner J, et al. Fate of pulmonary artery after anatomic correction of simple transposition of great arteries in newborn infants. Circulation 1988;78(4):870-876 